\title{
The role of recessive inheritance in early-onset epileptic encephalopathies: a combined whole-exome sequencing and copy number study
}

\author{
Sorina M. Papuc ${ }^{1,2} \cdot$ Lucia Abela $^{3,4,5} \cdot$ Katharina Steindl $^{1}$ - Anaïs Begemann $\mathbb{1}^{1} \cdot$ Thomas L. Simmons $^{3}$. \\ Bernhard Schmitt ${ }^{3,4}$ - Markus Zweier ${ }^{1}$ - Beatrice Oneda ${ }^{1}$ Eileen Socher ${ }^{6} \cdot$ Lisa M. Crowther $^{3} \cdot$ Gabriele Wohlrab $^{3}$. \\ Laura Gogoll ${ }^{1}$ Martin Poms ${ }^{3} \cdot$ Michelle Seiler $\mathbb{D}^{7} \cdot$ Michael Papik $^{1} \cdot$ Rosa Baldinger $^{1} \cdot$ Alessandra Baumer $^{1}$. \\ Reza Asadollahi ${ }^{1}{ }^{1}$ - Judith Kroell-Seger ${ }^{8}$ - Regula Schmid ${ }^{9} \cdot$ Tobias Iff $^{10}$ - Thomas Schmitt-Mechelke ${ }^{11}$. \\ Karoline Otten ${ }^{8} \cdot$ Annette Hackenberg $^{3} \cdot$ Marie-Claude Addor $^{12} \cdot$ Andrea Klein $^{13,14} \cdot$ Silvia Azzarello-Burri $^{1}$. \\ Heinrich Sticht ${ }^{6}$ - Pascal Joset ${ }^{1}$ - Barbara Plecko ${ }^{3,4,5,15,16}$ - Anita Rauch (iD) $1,5,15,17$
}

Received: 21 March 2018 / Revised: 5 October 2018 / Accepted: 25 October 2018 / Published online: 14 December 2018

(c) The Author(s) 2018. This article is published with open access

\begin{abstract}
Early-onset epileptic encephalopathy (EE) and combined developmental and epileptic encephalopathies (DEE) are clinically and genetically heterogeneous severely devastating conditions. Recent studies emphasized de novo variants as major underlying cause suggesting a generally low-recurrence risk. In order to better understand the full genetic landscape of EE and DEE, we performed high-resolution chromosomal microarray analysis in combination with whole-exome sequencing in 63 deeply phenotyped independent patients. After bioinformatic filtering for rare variants, diagnostic yield was improved for recessive disorders by manual data curation as well as molecular modeling of missense variants and untargeted plasmametabolomics in selected patients. In total, we yielded a diagnosis in $\sim 42 \%$ of cases with causative copy number variants in 6 patients $(\sim 10 \%)$ and causative sequence variants in 16 established disease genes in 20 patients $(\sim 32 \%)$, including compound heterozygosity for causative sequence and copy number variants in one patient. In total, $38 \%$ of diagnosed cases were caused by recessive genes, of which two cases escaped automatic calling due to one allele occurring de novo. Notably, we found the recessive gene SPATA5 causative in as much as $3 \%$ of our cohort, indicating that it may have been underdiagnosed in previous studies. We further support candidacy for neurodevelopmental disorders of four previously described genes (PIK3AP1, GTF3C3, UFC1, and WRAP53), three of which also followed a recessive inheritance pattern. Our results therefore confirm the importance of de novo causative gene variants in EE/DEE, but additionally illustrate the major role of mostly compound heterozygous or hemizygous recessive inheritance and consequently high-recurrence risk.
\end{abstract}

\section{Introduction}

The term epileptic encephalopathy (EE) denotes a rare group of severe, early-onset epilepsies that tend to be

These authors contributed equally: Sorina M. Papuc, Lucia Abela

These authors contributed equally: Barbara Plecko, Anita Rauch

Electronic supplementary material The online version of this article (https://doi.org/10.1038/s41431-018-0299-8) contains supplementary material, which is available to authorized users.

Anita Rauch

anita.rauch@medgen.uzh.ch

Extended author information available on the last page of the article pharmacoresistant with generally poor outcome and often severe intellectual disability [1]. EEs are heterogeneous with respect to seizure semiology and neurological comorbidities and encompass several clinical entities, such as Dravet- (MIM 607208), Ohtahara- (MIM 308350), and West syndromes (MIM 308350). They have been long thought to be acquired rather than genetic, given their common occurrence in a single individual with no family history [2]. Advances in genomic technologies over the past decade have unraveled a variety of genetic causes of epileptic encephalopathies and indicated that in some of these genetic entities intellectual disability or autism may be a primary clinical sign independent of the presence of epilepsy [1, 2]. These observations resulted in the new recommendation in 2017 to refer to EE only where "the 
abundant epileptiform activity interferes with development resulting in cognitive slowing and often regression" and "developmental and epileptic encephalopathy" (DEE) where there is additional developmental impairment independent of the epileptic activity [3].

Despite the growing number of known EE/DEE disease genes, the majority of patients still remain without etiological diagnosis. A retrospective, single-center cohort study evidenced that clinical signs, MRI, and targeted metabolic tests lead to an etiological diagnosis in about $13 \%$ of patients with "epileptic encephalopathy" [4]. In patients with a clinically unrecognized etiology (likely) causative de novo copy number variants (CNVs) accounted for about 3$10 \%$ of patients in several studies [4-8]. A large wholeexome sequencing study in "epileptic encephalopathy" patients established de novo variants, as disease cause in $12 \%$ [9]. This finding was further supported by gene panel and whole-genome sequencing studies which, considering those with a minimum of 50 patients, revealed a diagnostic yield of up to 37 and $32 \%$, respectively, again with the vast majority of disease-associated variants occurring as de novo events [4, 10-17]. However, a recent report on diagnostic exome sequencing in 89 unselected patients with "epileptic encephalopathy" yielded a diagnosis in $\sim 43 \%$ cases, including $\sim 10 \%$ with recessive inheritance [18]. In order to further evaluate the etiology and the role of recessive inheritance in EE/DEE, we performed a comprehensive single-center study to unravel the underlying etiology in 63 deeply phenotyped patients with early-onset EE or DEE of clinically unrecognized etiology by combining wholeexome sequencing and high-resolution chromosomal CNV studies.

\section{Subjects and methods}

\section{Patients}

After approval of this single-center study by the ethical committee of the Kanton of Zurich and following informed consent by the participating individuals or their legal guardians, patients, available parents, and selected further family members were enrolled from 2013-2015 if the patients fulfilled the following inclusion criteria: (1) developmental delay and onset of epilepsy below the age of 4.5 years (in order to encompass early-infantile, infantile, and early childhood-onset epilepsy), (2) pharmacoresistance for at least 6 months (reflecting the common definition of pharmacoresistance as ongoing seizures after trial of two different drugs and the time needed to try two antiepileptics), (3) no persistent spike wave focus in EEG (because they may indicate localized cortical dysplasia which is not yet detectable on early MR images as a distinct etiological clue), (4) absence of specific malformations on cerebral MRI, and (5) unknown etiology after standard clinical evaluation including an extended targeted metabolic screening (B6 vitamers, amino acids, and pipecolic acid in the plasma, alpha-aminoadipic semialdehyde and organic acids in urine). All patients were seen by experienced neuropediatricians and medical geneticists and deeply phenotyped including clinical and family history, physical examination and analysis of seizure semiology, and EEG findings.

\section{Chromosomal microarray analysis (CMA)}

After extraction of genomic DNA from peripheral blood samples, high-resolution chromosomal microarray studies were performed using Affymetrix Cytoscan HD arrays (containing about 750,000 genotype-able SNPs and 1.9 million non-polymorphic probes) (Affymetrix, Santa Clara, CA, USA), as previously described [19]. The analyses were primarily performed in patients only, followed by parental testing in case of (likely) causative or unclear findings in the patient. Rare CNVs not present in in-house and Affymetrix healthy control samples as well as CNVs overlapping recurrent disease loci underwent visual assessment of the actual marker location and distribution, and these were further investigated for their clinical relevance using the following databases: DECIPHER (https://decipher.sanger. ac.uk/), ISCA consortium (https://www.iscaconsortium.org/), OMIM (Online Mendelian Inheritance in Man, https://www.omim.org/), UCSC genome browser (http:// genome.ucsc.edu/), and PubMed (http://www.ncbi.nlm.nih. gov/pubmed). De novo CNVs were confirmed by alternative methods when their confidence score or marker content did not allow reliable calling of the CNV by array data alone. All detected rare CNVs were submitted to the DECIPHER database [20].

\section{Whole-exome sequencing and further studies}

Whole-exome sequencing was performed using the Agilent SureSelectXT Clinical Research Exome Kit (V5 CRE) (Agilent Technologies, Santa Clara, CA, USA) for capturing, followed by paired-end sequencing of $125 \mathrm{bp}$ forward and $125 \mathrm{bp}$ reverse using a HiSeq SBS Kit v4 on a HiSeq2500 System (Illumina, San Diego, CA, USA). To achieve a high average coverage, only $12-15$ samples were pooled within four lanes. Raw fastQ files were aligned to the hg19 reference genome using the NextGENe software (SoftGenetics, State College, PA, USA). Average sequencing coverage was 212 -fold with $>96 \%$ of the region-ofinterest covered at least 20 -fold. Variants were called if observed in at least $16 \%$ of reads with sufficient quality level. The resulting variants were annotated for potential 
damaging effects by standard in silico prediction tools, such as SIFT (http://sift.jcvi.org/), PolyPhen2 (http://genetics.bw h.harvard.edu/pph2/), LRT, MutationTaster (http://www. mutationtaster.org), MutationAssessor, FATHMM, GERP, and CADD (http://cadd.gs.washington.edu/), as provided within the NextGENe software. The ALAMUT visual prediction software version 2.7.2 (Interactive Biosoftware, Rouen, France) was used for additional exploration in selected variants. Rare protein changing variants and splice site variants (including 12 intronic basepairs) with an overall minor allele frequency below $2 \%$ were analyzed for de novo, compound heterozygous, and homozygous calls, and additionally for inherited X-chromosomal calls in boys, and visually inspected for verification of the assumed inheritance pattern. Resulting genes with potentially damaging variants were manually assessed for known or suspected association with EE or overlapping neurodevelopmental disorders using a variety of databases, such as ClinVar, DECIPHER, Exome Aggregation Consortium (ExAC, http://exac.broadinstitute.org/), HGMD (Human Genome Mutation Database, http://www.hgmd.org/), ISCA consortium, OMIM, UCSC genome browser, PubMed, Simons Foundation SFARI database (https://gene.sfari.org/), and SysID database (http://sysid.cmbi.umcn.nl/). All de novo variants, diagnostic and candidate disease variants were confirmed by Sanger sequencing and discussed within the team, including natural scientists, clinical geneticists, and neuropediatricians. In selected candidate missense variants, potential functional effects were further evaluated by structural mutation modeling. In patients with unavailability of one parent, in-silico designed filters for genes known to be associated with intellectual disability or epilepsy were used. Independently, all variants were filtered for overlap with specific variants described in the Human Genome Mutation Database, for inherited variants in known intellectual disability/epilepsy genes unreported at the time of analysis, and for loss-of-function variants in genes of the Human Genome Mutation Database. Classification of potentially deleterious variants in known disease genes followed the ACMG guidelines [21]. From the ExAC database, only variants that passed the quality filters were considered. In selected cases with only one diseaseassociated sequence or copy number variant in recessive disease genes, sequencing gaps were covered by Sanger sequencing. In selected cases with $\mathrm{X}$-chromosomal variants potentially affecting function, $\mathrm{X}$-inactivation ratios were tested as described by Lau et al. [22]. In selected cases with variants of unclear significance in splice sites or with monoallelic disease-associated variants in recessive disease genes RNA was extracted from blood samples using the PAXgene Blood System (PreAnalytiX, Hombrechtikon, Switzerland) and transcribed into cDNA using Superscript III Reverse Transcriptase and random hexamers (Invitrogen/
ThermoFisher Scientific, Waltham, MA, USA) and used for PCR amplifications and Sanger sequencing, respectively. All EE-associated and candidate variants were submitted to the LOVD [23] (Leiden Open Variation Database) accessible at www.LOVD.nl/ followed by the respective gene name, e.g., www.LOVD.nl/SPATA5 database. The respective LOVD patient IDs are given in Table 3 and the Supplementary Tables.

In plasma samples of three patients with findings from exome sequencing that indicated disturbance of metabolic pathways, we performed an untargeted metabolomic screen as previously described [24, 25].

\section{Results}

\section{Patient characteristics}

We recruited a total of 63 independent, mostly sporadic index cases with EE $(n=42 ; 67 \%)$ or DEE $(n=21 ; 33 \%)$. Five $(8 \%)$ had affected siblings: two had a likewise affected monozygotic twin, and three had one or two likewise affected siblings. Parents of four index cases $(6 \%)$ were consanguineous (three sporadic and one patient with affected siblings). In all but two families in whom the fathers were not available, DNA samples of both parents could be collected. Index cases comprised 38 males and 25 females. The median age of seizure onset was 7 months (range 1 month to $43 / 12$ years). The median age at last investigation of index patients was 7 years (range 6 months to 38 years). Four patients (6\%) deceased during the study. The 58 patients for whom detailed information was available took on average 5.6 different antiepileptic drugs over the course of their disease. Neurodevelopmental characteristics of the cohort are summarized in Table 1 and detailed phenotypic information is provided in Table S1 in the Supplementary Material.

\section{Genomic analyses}

Chromosomal microarray analysis revealed a total of 50 reliably called rare coding and recurrent disease loci CNVs in $33(52 \%)$ of index patients sizing $7-7700 \mathrm{~kb}$ with a median size of $110 \mathrm{~kb}$ and a maximum number of three per patient (Table S2 in the Supplementary Material). Six of the nine de novo CNVs affected well-known disease loci ( $\sim 10 \%$ of the cohort) (Fig. 1a and Tables $2-3$ and Tables S2 and S3 in the Supplementary Material). Five of the latter established a diagnosis unequivocally explaining the EE/ DEE phenotype, but we cannot exclude further contributing factors to pathogenicity in the de novo $2 \mathrm{Mb}$ duplication of the chromosomal region 22q11.22-22q11.23 (MIM 608363) representing a recurrent $\mathrm{CNV}$ between the low 
Table 1 Summary of main clinical characteristics in 63 index patients

\begin{tabular}{lc}
\hline Neurodevelopmental traits & $\begin{array}{l}\text { Number of patients } \\
\text { (percentage) }\end{array}$ \\
\hline Profound intellectual disability & $16(25.3 \%)$ \\
Moderate to severe intellectual & $47(74.6 \%)$ \\
disability & $21(33.3 \%)(44 \%$ of \\
Ataxia & those who could walk) \\
Cerebral palsy & $31(49.2 \%)$ \\
Muscular hypotonia & $49(77.8 \%)$ \\
Microcephaly & $27(41.9 \%)$ \\
Macrocephaly & $4(6.3 \%)$ \\
Single seizure type & $6(9.5 \%)$ \\
Multiple seizure types & $57(90.5 \%)$ \\
Status epilepticus & $13(20.6 \%)$ \\
Seizure Semiology & \\
Epileptic spasms & $20(31.8 \%)$ \\
Generalized/focal tonic seizures & $36(57 \%)$ \\
Generalized/focal clonic seizures & $10(15.9 \%)$ \\
Generalized tonic-clonic seizures & $33(52.4 \%)$ \\
Generalized/focal myoclonic & $31(49.2 \%)$ \\
seizures & $15(42.9 \%)$ \\
Atonic seizures & $5(7.9 \%)$ \\
Myoclonic-atonic seizures & $19(30.2 \%)$ \\
Focal seizures aware & $17(26.9 \%)$ \\
Focal seizures with impaired & $10(15.9 \%)$ \\
awareness & $7(11.1 \%)$ \\
Aarly-onset absences & \\
\hline &
\end{tabular}

copy repeats LCR22-E and LCR22-H causing a variable neurodevelopmental phenotype with reduced penetrance [26]. Four of the inherited heterozygous deletions affected known recessive disease genes, of which two, FARS2 (MIM 611592) and SPATA5 (MIM 613940), have been associated with neurodevelopmental disorders. Notably, one male and two female patients $(5 \%)$ without definite diagnosis harbored the recurrent Xp22.31 duplication or triplication of $1.7 \mathrm{Mb}$ including the STS (MIM 300747) locus, which has been discussed as a risk factor for neurodevelopmental disease and epilepsy in both sexes [27-29]. In our Affymetrix European and American healthy control cohort, the duplication was found in three females out of 1038 individuals indicating a population prevalence of $0.3 \%$, which is similar to that found in a previous study [29]. While the latter study found no significant difference between 20,095 various clinical cases and 5088 controls, the frequency of copy number gains at the STS locus was significantly increased in our epileptic encephalopathy cohort $(p<$ 0.0001, Chi-Square two-sided test). We therefore analyzed further available members in two families, which indicated no segregation of the duplication with the phenotype, but left the clinical relevance of the triplication open (Supplemental Figure S1 and Table S2 in the Supplementary Material).

Whole-exome sequencing was performed in 60 patients, one sibling and 104 parents. Three of the five patients with a de novo CNV fully explaining the phenotype were not exome sequenced. In nine patients, only the index cases were exome sequenced and since a final diagnosis could be already reached in eight of these, parental samples were only used for segregation analysis by Sanger sequencing. Since only one patient in whom trio-sequencing was not possible due to lack of parental samples remained without diagnosis, this approach did not affect diagnostic yield statistics. In the 51 trios who underwent whole-exome sequencing in the patients and both parents we identified per patient a median of one de novo, one homozygous, seven compound heterozygous, and nine $\mathrm{X}$-linked inherited rare variants (Fig. 1b). In 20 patients, disease-associated variants in established $\mathrm{EE} / \mathrm{DEE}$ genes were identified establishing an etiological diagnosis in $32 \%$ of the total cohort (Fig. 1c, d, Table 3, Tables S3 and S4 in the Supplementary Material). These sequence alterations include 15 novel variants, first described in this paper, and occurred in 14 well-known EE/DEE genes as well as in two disease genes (GABRB2 [MIM 600232], PRUNE1 [MIM 617413]) previously reported in a few patients only (Table 2 and S3). Very recently, Hamdan et al. [16] reported 11 patients harboring de novo missense variants in GABRB2 with early-onset epilepsy, severe intellectual disability, acquired microcephaly, hypotonia, and further neurological signs in the majority of patients. Our patient (ID 73324) harboring a novel de novo variant c.719G $>$ C p.(Arg240Thr) in $G A B R B 2$ likely affecting function also shows these common features (see Table S1 and S3 in the Supplementary Material).

PRUNE1 was recently described to cause a novel autosomal recessive disease (MIM 617481) characterized by severe global developmental delay, microcephaly, dysmorphic features, muscle tone abnormalities, and variable brain abnormalities such as delayed myelination, thin corpus callosum or cerebellar hypoplasia, but several patients are also reported to have early-onset seizures (see also Supplementary Table S8) [30]. Our index patient (ID 69937) from consanguineous parents had a severe DEE phenotype but cerebral MRI showed no malformation but rather immature cortex differentiation and discrete signs of hypoxia. He had two brothers with a similar phenotype that died at the age of $4 \mathrm{~m}$ and 2 y $9 \mathrm{~m}$, respectively (see Table S1 in the Supplementary Material). The homozygous variant in PRUNE1 (NM_021222.2:c.[316 G > A];[316 G > A] p.[(Asp106Asn)];[(Asp106Asn)]), details in Table S3 in the Supplementary Material) detected in 
A Percentage of patients with rare CNVs

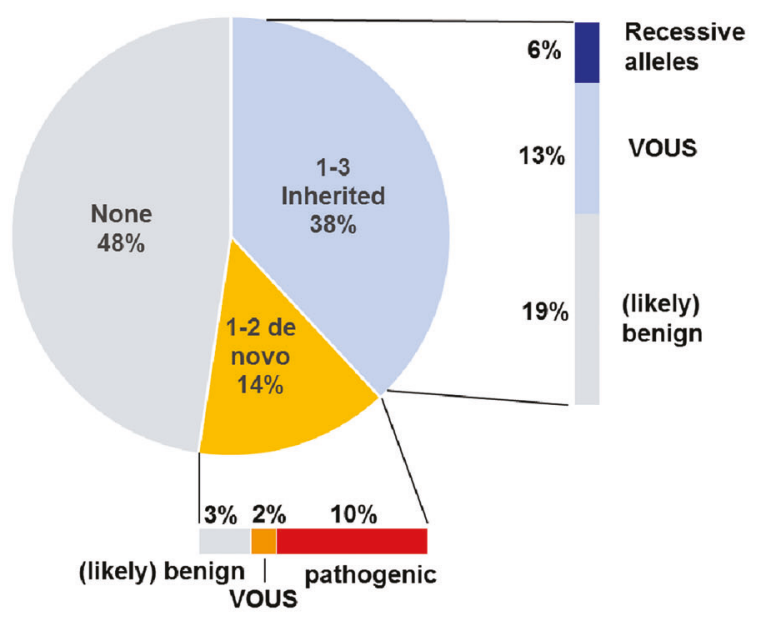

C Percentage of patients with variants associated with EE or candidate variants

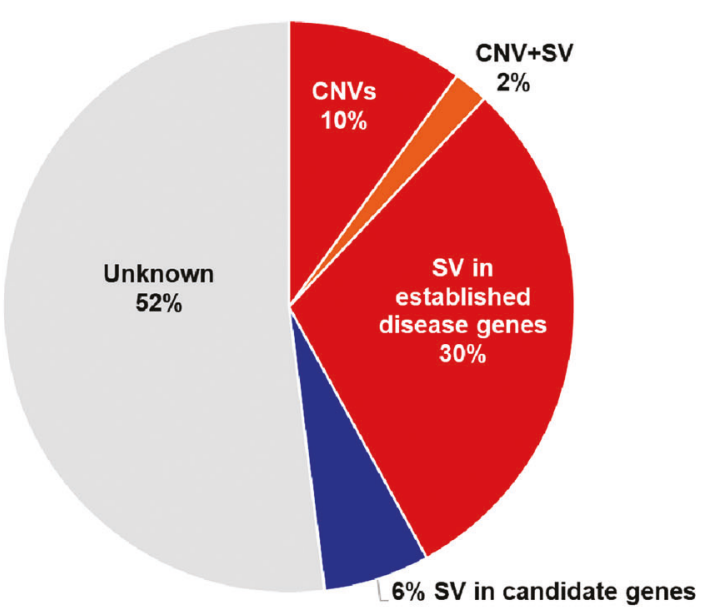

E Classification of secondary sequence variants in known disease genes

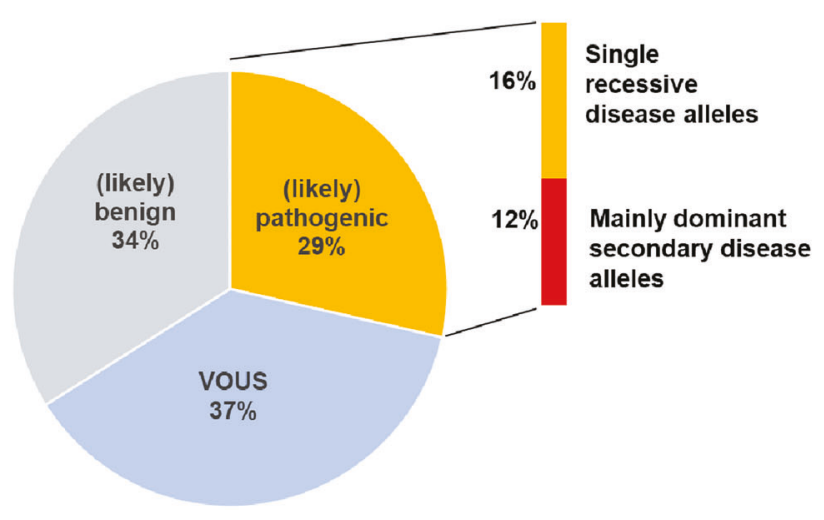

B Minimum, maximum and median number of rare sequence variants per patient following the respective inheritance pattern

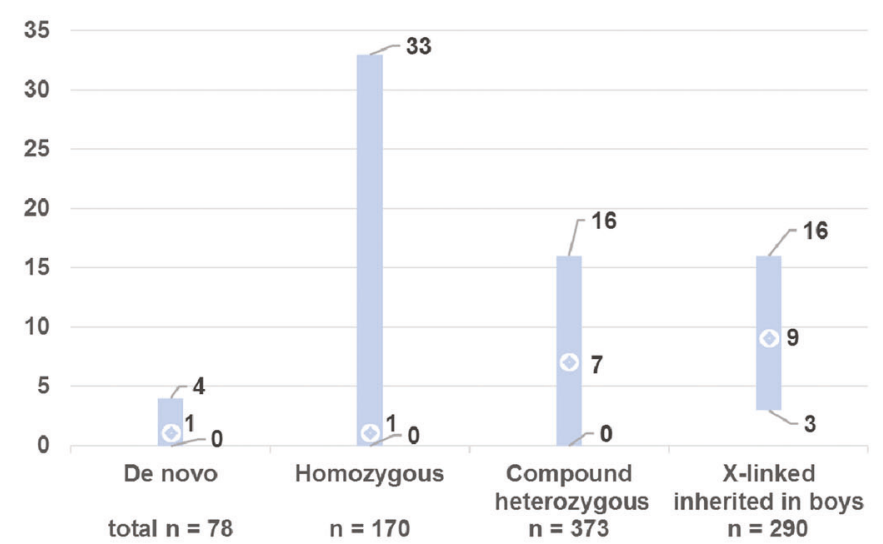

D Mode of inheritance in diagnosed patients

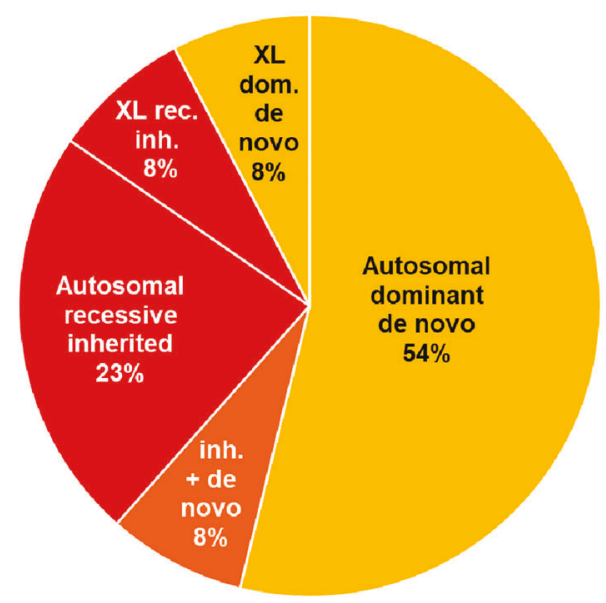

our patient and his available affected brother is the same sequence variant that was already reported as diseaseassociated in two families by Karaca et al. [30].
In two index patients and one affected brother with variants of unclear significance in established disease genes affecting metabolic pathways, we were able to confirm 
4 Fig. 1 Summary of genetic findings. a Percentage of patients with $\geq 1$ de novo or inherited rare copy number variants and their respective classification of clinical relevance. b Total numbers, and medians, minimum and maximum numbers per patients of variants following the respective modes of inheritance identified by whole-exome sequencing in 51 child-parent trios. c Percentage of patients with (likely) disease-associated $\mathrm{CNV}$ or SV findings in established EE genes or with potentially disease-causing candidate genes. d Distribution of inheritance modes in diagnosed patients with (likely) EEassociated CNV or SV in established disease genes. e Results of (re-) classification of 56 secondary findings from whole-exome sequencing, of which $16 \%$ represented carriership of single alleles of recessive disorders and $12 \%$ indicated mainly dominant disease alleles as secondary findings. CNV copy number variant, SV sequence variant, VOUS variants of unknown clinical significance, XL dom. X-linked dominant, XL rec. inh. X-linked recessive inherited

pathogenicity by untargeted metabolomic analyses. In monozygotic twin brothers (ID 72555 and 72719) carrying a missense variant in the X-linked spermine synthase gene (SMS, MIM 300105) related to Snyder-Robinson syndrome, we found significantly increased N8acetylspermidine in plasma, a metabolite that accumulates upstream to the enzyme defect [25]. In a female patient (ID 69986) with two novel inherited compound, heterozygous variants in the aconitase 2 gene (ACO2, MIM 100850) plasma metabolomic analysis distinguished a metabolic fingerprint profile that reflects the enzyme deficiency within the citric acid cycle but also perturbations in associated metabolic pathways [24].

Additionally, we searched for secondary findings in the exome sequencing data and hereby identified 56 specifically reported disease alleles or loss-of-function or unreported variants in Human Genome Mutation Database genes not related to EE and reclassified them manually (Fig. 1e and Table 3 and S7 of the Supplementary Material). An overview of primary and secondary findings, as well as variants in candidate genes is provided in Table S8 of the Supplementary Material.

\section{Discussion}

Applying high-resolution chromosomal copy number profiling and whole-exome sequencing in 63 index cases with EE/DEE and the majority of their healthy parents established a diagnosis in $\sim 42 \%$ of patients. De novo CNVs accounted for $\sim 10 \%$ of the diagnostic yield, which is higher than the 3-5\% reported in most of previous studies [4-7], but is in line with a recent report on copy number profiling from exome sequencing data [8] and may be explained by the higher genome-wide resolution for CNV detection of our high-resolution chromosomal microarray analysis in comparison with the lower-resolution microarrays and exome sequencing data previously used. Although CNVs might be identified by read-depth analysis from exome sequencing data with a similar diagnostic yield as by highresolution microarrays, high-resolution copy-number profiling from exome data requires significantly more confirmatory work-up owing to their lower specificity [31]. Our $\sim 32 \%$ diagnostic yield achieved by whole-exome sequencing is within the range published by previous studies, but due to varying pretesting, patient inclusion criteria and cohort compositions direct comparison is difficult [4, 9-16, 18, 32-34]. Nevertheless, with respect to our $\sim 16 \%$ diagnostic yield from causative de novo sequence variants, which comprise the vast majority in the latter studies, we found the same spectrum of affected genes. As a major difference, however, we additionally found (likely) diseaseassociated variants in autosomal and $\mathrm{X}$-linked recessive genes in another $\sim 16 \%$ of index patients. This finding is not explained by consanguinity, since only one of ten of these index patients is an offspring of a consanguineous couple. There is also neither a difference in the ratio of EE/DEE diagnoses and age of onset of seizures, nor any other apparent clinical difference between the dominant and recessive disease group. The only previous report of a considerable diagnostic yield of $11 \%$ for recessive sequence variants so far was a diagnostic whole-exome sequencing study with limited clinical data [18]. Given the high number of rare inherited variants per patient (Fig. 1b), identification of causative inherited pathogenic variants is much more challenging than the evaluation of de novo variants, and in our study required extensive manual data annotation including interdisciplinary clinical assessment, a comprehensive search for second hits in single-recessive variants as well as functional evidence for pathogenicity of missense variants derived from structural mutation modeling and untargeted metabolome data. Notably, metabolomic analysis increased our diagnostic yield for recessive disease genes by supporting the pathogenicity of missense variants involved in metabolic pathways, as illustrated for Snyder Robinson syndrome and ACO2 deficiency [24, 25]. Moreover, in three cases with biallelic disease-associated variants in known autosomal recessive disease genes, only manual curation unraveled the respective second variant. In these three cases, there was a single-inherited sequence variant in trans with either a de novo sequence or an inherited copy number variant each (Fig. 2). Accordingly, the singleinherited sequence variants were initially filtered out by standard algorithms, since compound heterozygosity is commonly defined as two variants occurring at different genomic positions within the same gene with one variant inherited from each heterozygous parent [35]. As no previous study considered copy-number and sequence variants for compound heterozygous hits, such allele constellations were not called by filtering strategies reported so far. Without these three challenging cases, our diagnostic yield 
Table 2 Summary of established genes causing EE/DEE in our study cohort, as well as recurrent candidate genes for neurodevelopmental disorders

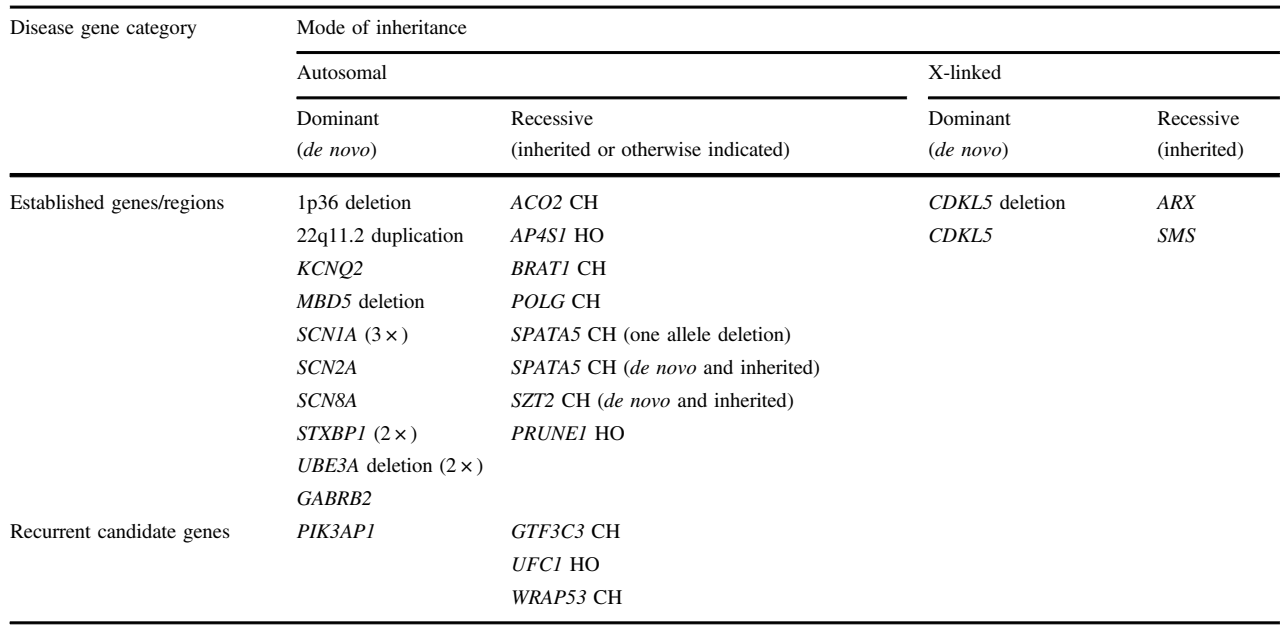

$\mathrm{CH}$ compound heterozygous, $\mathrm{HO}$ homozygous for recessive disease genes would be in the same range as reported by Helbig et al. [18]. These unexpected mechanisms may also explain why we found SPATA5, which was rarely reported so far [36-40], as a more common cause of EE/DEE $(2 / 63 ; 3 \%)$. In order to independently prove this high frequency of SPATA5-related EE and DEE, we analyzed its prevalence in 39 cases with infantile or childhood epilepsies who received diagnostic exome sequencing and identified one case carrying a previously reported homozygous SPATA5 disease-associated variant NM_145207.2 (SPATA5):c.[251 G > A];[251 G > A] p.[(Arg84Gln)]; [(Arg84Gln)]. This diagnostic case with developmental encephalopathy with late-onset epilepsy also had an unusual mutational mechanism with homozygotization of a maternally inherited variant due to maternal isodisomy of chromosome 4. Thus, our overall finding of 3 out of 102 combined research and diagnostic patients carrying causative biallelic variants indicates that SPATA5 is a frequent cause of developmental epileptic disorders accounting for $3 \%$ of cases (Table 4). Notably, in a recently published whole-exome sequencing study of 320 patient-parent trios with EE a single patient with compound heterozygous SPATA5 pathogenic variants was the only case with autosomal recessive disease cause found [33].

Concerning the broad variant filtering applied (minor allele frequency (MAF) $<2 \%,+/-12$ flanking intronic bp, $16 \%$ aberrant allele frequency), our findings of three EEassociated variants within three to six flanking intronic basepairs indicate the importance of evaluation of the splicing region beyond the canonical splice site. However, the highest European MAF observed amongst our EEassociated variants was $0.03 \%$ (SPATA5 rs748291365) for recessive disease genes, and one de novo variant (SCN8A rs796053229) had a MAF of $0.003 \%$ in the Latino population and an overall MAF of $0.0004 \%$ in gnomAD. With reference to the aberrant allele frequency, the lowest observed in our cohort was $32 \%$ in apparently heterozygous inherited disease-associated variants, but we did not identify a de novo variant below this value indicative of a mosaic status.

It is also noteworthy that our findings further support the disease association of the two recently established disease genes GABRB2 and PRUNE1, as well as the candidacy of PIK3AP1, GTF3C3, WRAP53, and UFC1 as potential neurodevelopmental disease genes (see Table S5 in the Supplementary Material). To date, only two de novo missense variants in PIK3API have been reported in two independent patients with infantile spasms [41]. Our patient harbors an unreported de novo missense variant with deleterious predictions in the DBB domain, which is required in other proteins to mediate protein-protein interaction. Further reports and functional studies are needed to evaluate the potential role of this gene in neurodevelopmental disorders.

GTF3C3 was classified as moderately confident candidate gene in a recently published study on neurodevelopmental disorders in consanguineous families after detection of homozygous missense variants in two affected sisters with a phenotype including mild ID, seizures, and dysmorphism [42]. Another study reported a patient with profound microcephaly, facial dysmorphism, and failure to thrive to harbor a homozygous splice site variant in GTF3C3 causing skipping of exon 10 and parts of exon 11 (in-frame deletion) [43]. Furthermore, the yeast ortholog of GTF3C3, Tfc4, has been shown to interact with BRF1 to regulate RNA polymerase III-mediated transcription [44, 45]. Biallelic variants in BRFI (MIM 604902) cause the cerebellofaciodental syndrome (MIM 616202) with a similar phenotype as our patient (delayed development, intellectual disability, abnormal facial and dental findings, and cerebellar hypoplasia) [46, 47]. Thus, we consider GTF3C3 as a candidate for neurodevelopmental disorders which may or may not include epilepsy. 







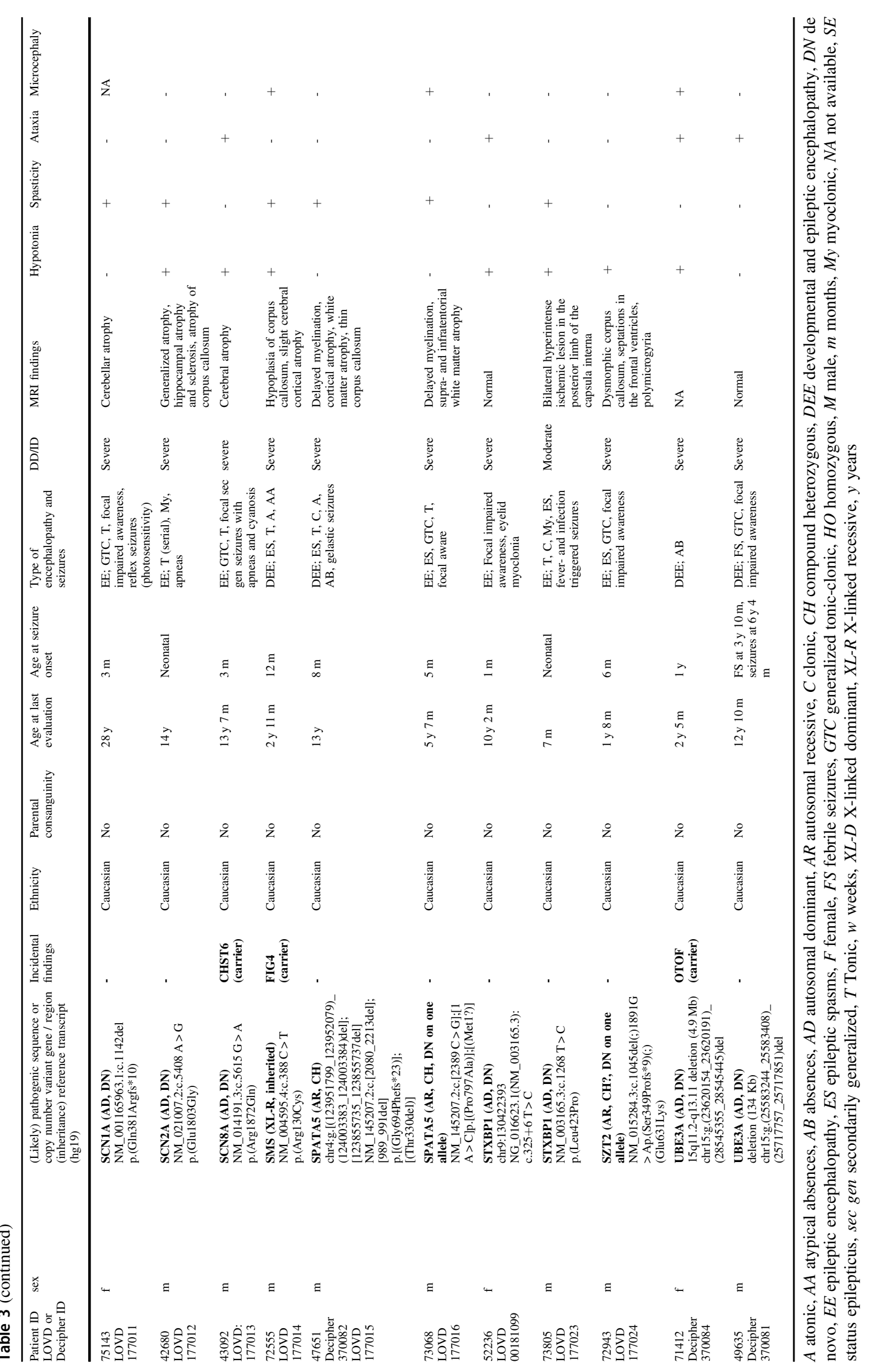


A

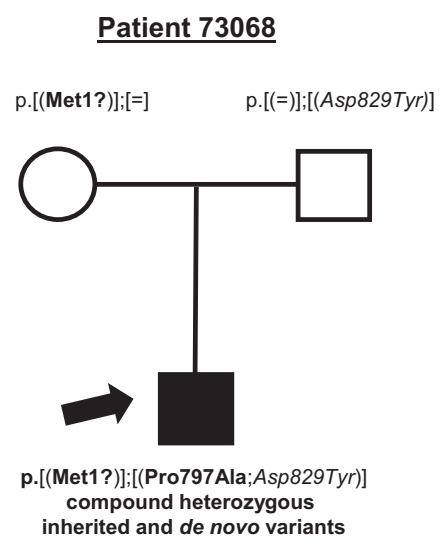

inherited and de novo variants
B

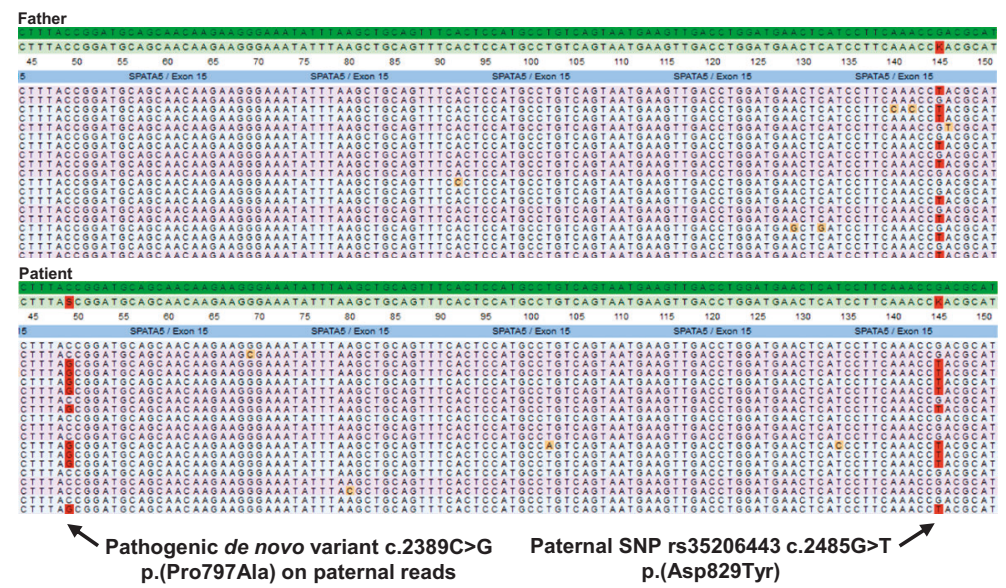

D

$51 \mathrm{~kb}$ deletion including exons $12 \& 13$ (c.2080_2213del, p.(Gly694Phefs*23))
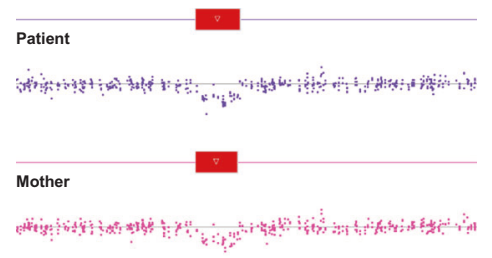

Father

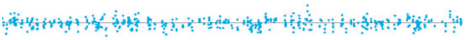

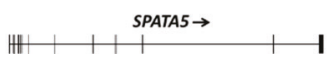

E c. 989_991del, p.(Thr330del)

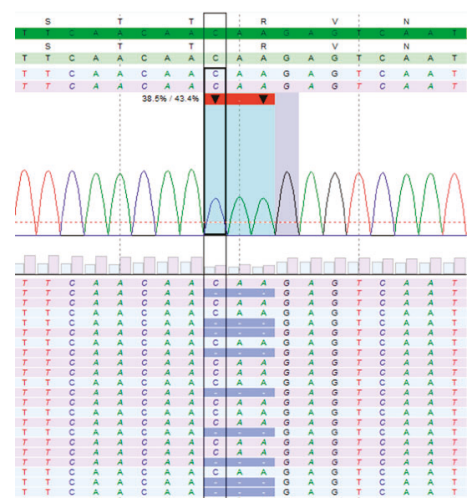

Fig. 2 Disease causing biallelic SPATA5 variants in two independent families demonstrating the value of hypothesis driven manual data analysis (variant nomenclature and exon numbering according to NM_145207.2 and NG_051570.1, respectively). a, b Pedigree of patient 73068 (a) summarizing the segregation of the maternally inherited variant p.(Met1?) and the de novo variant p.(Pro797Ala) on the paternal allele; (b) Whole-exome sequencing data of exon 15 in the patient demonstrating the localization of both, the de novo variant c. $2389 \mathrm{C}>\mathrm{G}$ (p.(Pro797Ala)) and the paternally inherited single nucleotide polymorphism (SNP) rs35206443 (c.2485 G>T, p. (Asp829Tyr)) on the same allele. c-e Pedigree of patient 47651 illustrating the compound heterozygous constellation of the maternally inherited, heterozygous $51 \mathrm{~kb}$ deletion comprising exon 12 and 13 (c.2080_2213del; p.(Gly694Phefs*23)) detected by high-resolution chromosomal microarray analysis (d), and the paternally inherited disease-associated sequence variant c.989_991del (p.(Thr330del)) detected by whole-exome sequencing (e)
Autosomal-recessive variants in WRAP53 have been reported as disease cause in two unrelated patients with the classical phenotype of Dyskeratosis congenita (DC) (MIM 613988) [48], supported by functional studies of the detected variants [48]. Our patient harbors two variants in compoundheterozygous constellation, NM_018081.2:c.[1034 A > G]; [1303 G > A] p.[(Tyr345Cys)];[[(Gly435Arg)] (see Table S5 in the Supplementary Material), of which the first is novel and the latter has been reported and functionally studied by Zhong et al. [48]. As previously described in other DC subtypes [49], our $2 \frac{1}{2}$ year-old patient shows delayed development, intrauterine growth retardation, cerebellar hypoplasia, microcephaly, sparse fine hair, and bone marrow failure, but does not yet present classical signs of variable age of onset, such as nails dysplasia, abnormal pigmentation, and oral leukoplakia.
Additionally, our patient has early onset and therapy resistant absence seizures, which is in line with findings in a DC mouse model [50], but this expansion of the phenotype needs confirmation by further observations.

UFC1 was first proposed as a candidate gene for recessive ID after the detection of a homozygous missense variant in two siblings with global developmental delay and microcephaly by Anazi et al. [51]. A collaborative effort on this gene revealed in addition to our patient with a homozygous missense variant further five patients from two families harboring a distinct homozygous variant, all sharing a phenotype of early-onset encephalopathy (microcephaly, short stature, global developmental delay, seizures, failure to thrive). This cohort was recently published along with evidence for a hypomorphic effect of both missense 


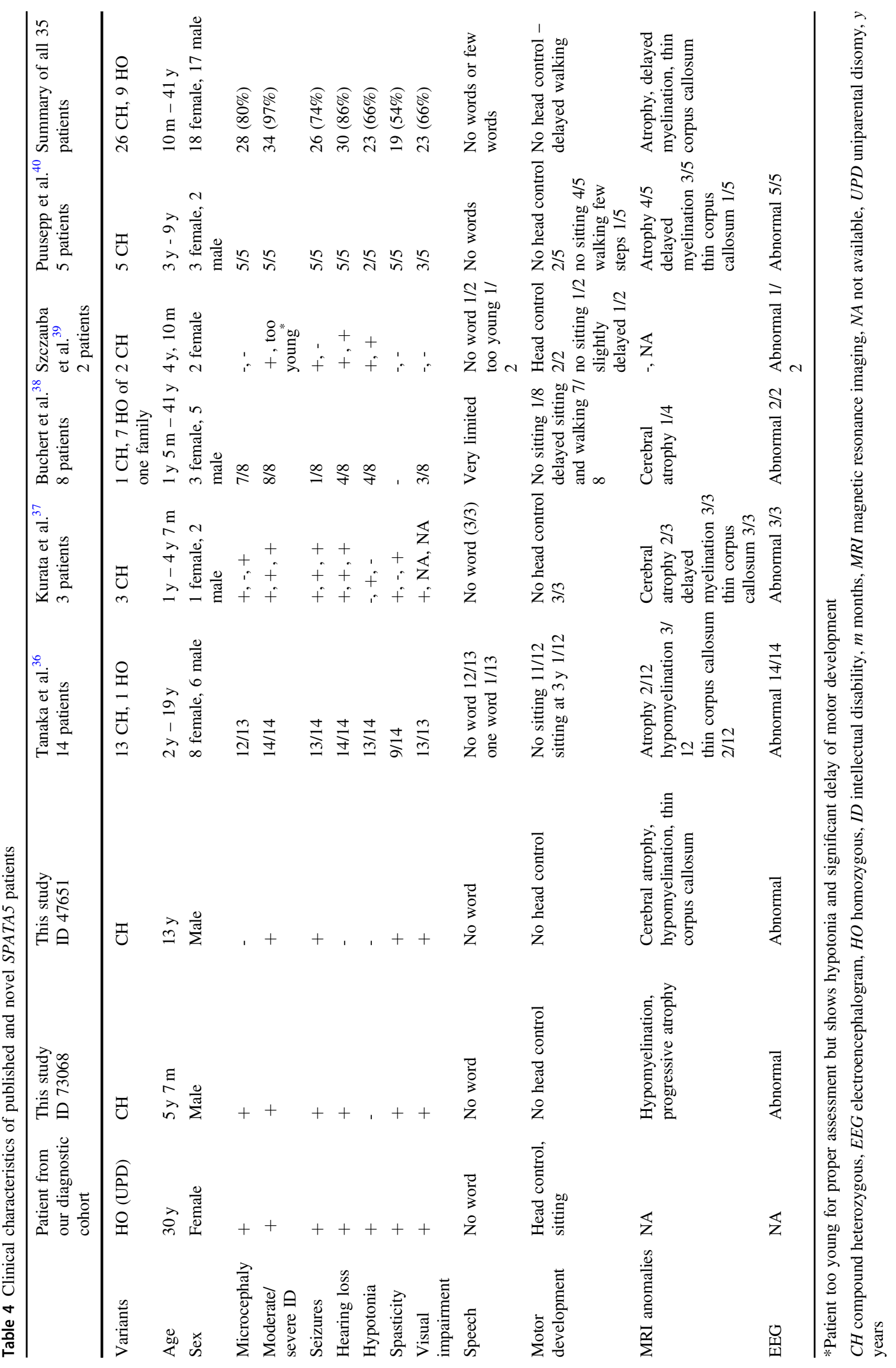


variants in functional studies [52]. Of note, variants in $U B A 5$ and $U F M 1$ in the same ufmylation pathway have also been described in patients with early-onset encephalopathy [52-56].

With respect to genetic counseling, autosomal or Xlinked recessive disorders were identified in $38 \%$ of diagnosed cases. Since in two cases with autosomal recessive variants one allele each occurred as de novo alteration, a high-recurrence risk of $25 \%$ for further siblings was present in $31 \%$ of the diagnosed cases. In contrast to previous assumptions [2], this high-recurrence risk is not restricted to offspring of consanguineous parents, which accounted for a small minority of our cases with recessive inheritance. Thus, the true recurrence risk may have been underestimated due to the low number of pregnancies following such severely affected patients in the hitherto absence of prenatal diagnostic options. Accordingly, as a direct result from the identification of the underlying genetic cause, $19 \%$ of all diagnosed families benefited from targeted prenatal testing during this study. Moreover, the genetic diagnosis led to a change in epilepsy treatment in $12 \%$ of diagnosed patients. In line with a previous report indicating a higher diagnostic yield of 39\% de novo pathogenic variants in very early-onset epilepsy ( $\leq 2$ months) in comparison with seizure disorders in general (18\%) [57], we found a remarkably high-diagnostic yield of $77 \%$ de novo and recessive pathogenic variants in patients with seizure onset within the first 2 months in comparison with our whole cohort $(\sim 42 \%)$.

We also revealed additional genetic disorders as secondary findings in known disease genes in $10 \%$ of patients (Asthenozoospermia, late-onset mild hearing impairment, predisposition to endocrine tumors and adrenal Cushing syndrome, Noonan syndrome 5, left ventricular noncompaction 7, MPO deficiency; Supplementary Table S8). This observation of blended phenotypes is higher than in a broader clinical exome sequencing study [58], but similar to the results of a whole-exome sequencing study in patients with combined phenotypes of metabolic and intellectual developmental disorders [59].

In conclusion, we found evidence of causative copy number and sequence variants in established disease genes explaining together with recurrent candidate genes about $48 \%$ of patients with EE/DEE with an unexpected high contribution of inherited recessive disorders, which is neither explained by founder mutations nor by consanguinity, imposing a significant recurrence risk even in outbred populations.

Acknowledgements This study was supported by radiz-Rare Disease Initiative Zürich, Clinical Research Priority Program for Rare Diseases of the University of Zurich to Drs. Lucia Abela, Barbara Plecko und Anita Rauch, by the University of Zurich Filling the Gap program to Dr. Lucia Abela, by the Scientific Exchange Programme between
Switzerland and the novel member states of the EU (Sciex-NMSch project code 12.351) to Drs. Sorina M. Papuc and Anita Rauch, and by the Forschungskredit UZH and the Josef Huwyler Ruth Bernet-Engeli Stiftung to Dr. Anaïs Begemann.

\section{Compliance with ethical standards}

Conflict of interest The authors declare that they have no conflict of interest.

Open Access This article is licensed under a Creative Commons Attribution 4.0 International License, which permits use, sharing, adaptation, distribution and reproduction in any medium or format, as long as you give appropriate credit to the original author(s) and the source, provide a link to the Creative Commons license, and indicate if changes were made. The images or other third party material in this article are included in the article's Creative Commons license, unless indicated otherwise in a credit line to the material. If material is not included in the article's Creative Commons license and your intended use is not permitted by statutory regulation or exceeds the permitted use, you will need to obtain permission directly from the copyright holder. To view a copy of this license, visit http://creativecommons. org/licenses/by/4.0/.

\section{References}

1. Berg AT, Berkovic SF, Brodie MJ, Buchhalter J, Cross JH, van Emde Boas W, et al. Revised terminology and concepts for organization of seizures and epilepsies: report of the ILAE commission on classification and terminology, 2005-2009. Epilepsia. 2010;51:676-85.

2. Myers CT, Mefford HC. Genetic investigations of the epileptic encephalopathies: recent advances. Prog Brain Res. 2016;226:3560 .

3. Scheffer IE, Berkovic S, Capovilla G, Connolly MB, French J, Guilhoto L, et al. ILAE classification of the epilepsies: position paper of the ILAE commission for classification and terminology. Epilepsia. 2017;58:512-21.

4. Mercimek-Mahmutoglu S, Patel J, Cordeiro D, Hewson S, Callen D, Donner EJ, et al. Diagnostic yield of genetic testing in epileptic encephalopathy in childhood. Epilepsia. 2015;56:707-16.

5. Mefford HC, Yendle SC, Hsu C, Cook J, Geraghty E, McMahon $\mathrm{JM}$, et al. Rare copy number variants are an important cause of epileptic encephalopathies. Ann Neurol. 2011;70:974-85.

6. Ma Y, Chen C, Wang Y, Wu L, He F, Chen C, et al. Analysis copy number variation of Chinese children in early-onset epileptic encephalopathies with unknown cause. Clin Genet. 2016;90:42836.

7. Epilepsy Phenome/Genome Project Epi KC. Copy number variant analysis from exome data in 349 patients with epileptic encephalopathy. Ann Neurol. 2015;78:323-8.

8. Tsuchida N, Nakashima M, Kato M, Heyman E, Inui T, Haginoya $\mathrm{K}$, et al. Detection of copy number variations in epilepsy using exome data. Clin Genet. 2018;93:577-87.

9. Euro E-RESC, Epilepsy Phenome/Genome P, Epi KC. De novo mutations in synaptic transmission genes including DNM1 cause epileptic encephalopathies. Am J Hum Genet. 2014;95: 360-70.

10. Kodera H, Kato M, Nord AS, Walsh T, Lee M, Yamanaka G, et al. Targeted capture and sequencing for detection of mutations causing early onset epileptic encephalopathy. Epilepsia. 2013;54:1262-9.

11. de Kovel CG, Brilstra EH, van Kempen MJ, Van't Slot R, Nijman IJ, Afawi Z, et al. Targeted sequencing of 351 candidate genes for 
epileptic encephalopathy in a large cohort of patients. Mol Genet Genom Med. 2016;4:568-80.

12. Carvill GL, Heavin SB, Yendle SC, McMahon JM, O'Roak BJ, Cook J, et al. Targeted resequencing in epileptic encephalopathies identifies de novo mutations in CHD2 and SYNGAP1. Nat Genet. 2013;45:825-30.

13. Arafat A, Jing P, Ma Y, Pu M, Nan G, Fang H, et al. Unexplained early infantile epileptic encephalopathy in Han Chinese children: next-generation sequencing and phenotype enriching. Sci Rep. 2017;7:46227.

14. Allen NM, Conroy J, Shahwan A, Lynch B, Correa RG, Pena SD, et al. Unexplained early onset epileptic encephalopathy: Exome screening and phenotype expansion. Epilepsia. 2016;57:e12-17.

15. Segal E, Pedro H, Valdez-Gonzalez K, Parisotto S, Gliksman F, Thompson S, et al. Diagnostic yield of epilepsy panels in children with medication-refractory epilepsy. Pediatr Neurol. 2016;64:6671 .

16. Hamdan FF, Myers CT, Cossette P, Lemay P, Spiegelman D, Laporte $\mathrm{AD}$, et al. High rate of recurrent de novo mutations in developmental and epileptic encephalopathies. Am J Hum Genet. 2017;101:664-85.

17. Ko A, Youn SE, Kim SH, Lee JS, Kim S, Choi JR, et al. Targeted gene panel and genotype-phenotype correlation in children with developmental and epileptic encephalopathy. Epilepsy Res. 2018;141:48-55.

18. Helbig KL, Farwell Hagman KD, Shinde DN, Mroske C, Powis Z, $\mathrm{Li} \mathrm{S}$, et al. Diagnostic exome sequencing provides a molecular diagnosis for a significant proportion of patients with epilepsy. Genet Med. 2016;18:898-905.

19. Asadollahi R, Oneda B, Joset P, Azzarello-Burri S, Bartholdi D, Steindl K, et al. The clinical significance of small copy number variants in neurodevelopmental disorders. $\mathrm{J}$ Med Genet. 2014;51:677-88.

20. Firth HV, Richards SM, Bevan AP, Clayton S, Corpas M, Rajan $D$, et al. DECIPHER: database of chromosomal imbalance and phenotype in humans using ensembl resources. Am J Hum Genet. 2009;84:524-33.

21. Richards S, Aziz N, Bale S, Bick D, Das S, Gastier-Foster J, et al. Standards and guidelines for the interpretation of sequence variants: a joint consensus recommendation of the American College of Medical Genetics and Genomics and the Association for Molecular Pathology. Genet Med. 2015;17:405-24.

22. Lau AW, Brown CJ, Penaherrera M, Langlois S, Kalousek DK, Robinson WP. Skewed X-chromosome inactivation is common in fetuses or newborns associated with confined placental mosaicism. Am J Hum Genet. 1997;61:1353-61.

23. Fokkema IF, Taschner PE, Schaafsma GC, Celli J, Laros JF, den Dunnen JT. LOVD v.2.0: the next generation in gene variant databases. Hum Mutat. 2011;32:557-63.

24. Abela L, Spiegel R, Crowther L, Klein A, Steindl K, Papuc SM, et al. Plasma metabolomics reveals a diagnostic metabolic fingerprint for mitochondrial aconitase (ACO2) deficiency. PLoS ONE. 2017;12:e0176363.

25. Abela L, Simmons L, Steindl K, Schmitt B, Mastrangelo M, Joset $\mathrm{P}$, et al. N(8)-acetylspermidine as a potential plasma biomarker for Snyder-Robinson syndrome identified by clinical metabolomics. J Inherit Metab Dis. 2016;39:131-7.

26. Wincent J, Bruno DL, van Bon BW, Bremer A, Stewart H, Bongers EM, et al. Sixteen new cases contributing to the characterization of patients with distal 22q11.2 microduplications. Mol Syndromol. 2010;1:246-54.

27. Addis L, Rosch RE, Valentin A, Makoff A, Robinson R, Everett $\mathrm{KV}$, et al. Analysis of rare copy number variation in absence epilepsies. Neurol Genet. 2016;2:e56.

28. Esplin ED, Li B, Slavotinek A, Novelli A, Battaglia A, Clark R, et al. Nine patients with Xp22.31 microduplication, cognitive deficits, seizures, and talipes anomalies. Am J Med Genet A. 2014;164A:2097-103.

29. Liu P, Erez A, Nagamani SC, Bi W, Carvalho CM, Simmons AD, et al. Copy number gain at $\mathrm{Xp} 22.31$ includes complex duplication rearrangements and recurrent triplications. Hum Mol Genet. 2011;20:1975-88.

30. Karaca E, Harel T, Pehlivan D, Jhangiani SN, Gambin T, Coban Akdemir Z, et al. Genes that affect brain structure and function identified by rare variant analyses of mendelian neurologic disease. Neuron. 2015;88:499-513.

31. Pfundt R, Del Rosario M, Vissers L, Kwint MP, Janssen IM, de Leeuw $\mathrm{N}$, et al. Detection of clinically relevant copy-number variants by exome sequencing in a large cohort of genetic disorders. Genet Med. 2017;19:667-75.

32. Epi KC. Epilepsy Phenome/Genome P, Allen AS et al. De novo mutations in epileptic encephalopathies. Nature. 2013;501:21721.

33. Epi KC. Euro E-RESC, Epilepsy Phenome Genome P. Application of rare variant transmission disequilibrium tests to epileptic encephalopathy trio sequence data. Eur J Hum Genet. 2017;25:894-9.

34. Zhou P, He N, Zhang JW, Lin ZJ, Wang J, Yan LM, et al. Novel mutations and phenotypes of epilepsy-associated genes in epileptic encephalopathies. Genes Brain Behav. 2018;17: e12456.

35. Zhu X, Petrovski S, Xie P, Ruzzo EK, Lu YF, McSweeney KM, et al. Whole-exome sequencing in undiagnosed genetic diseases: interpreting 119 trios. Genet Med. 2015;17:774-81.

36. Tanaka AJ, Cho MT, Millan F, Juusola J, Retterer K, Joshi C, et al. Mutations in SPATA5 are associated with microcephaly, intellectual disability, seizures, and hearing loss. Am J Hum Genet. 2015;97:457-64.

37. Kurata H, Terashima H, Nakashima M, Okazaki T, Matsumura W, Ohno K, et al. Characterization of SPATA5-related encephalopathy in early childhood. Clin Genet. 2016;90:437-44.

38. Buchert R, Nesbitt AI, Tawamie H, Krantz ID, Medne L, Helbig I, et al. SPATA5 mutations cause a distinct autosomal recessive phenotype of intellectual disability, hypotonia and hearing loss. Orphanet J Rare Dis. 2016;11:130.

39. Szczaluba K, Szymanska K, Kosinska J, Pollak A, Murcia V, Kedra A, et al. Isolated hearing impairment caused by SPATA5 Mutations in a family with variable phenotypic expression. Adv Exp Med Biol. 2017;980:59-66.

40. Puusepp S, Kovacs-Nagy R, Alhaddad B, Braunisch M, Hoffmann GF, Kotzaeridou U, et al. Compound heterozygous SPATA5 variants in four families and functional studies of SPATA5 deficiency. Eur J Hum Genet. 2018;26:407-19.

41. EuroEPINOMICS-RES Consortium. Epilepsy Phenome/Genome Project; Epi4K Consortium. De novo mutations in synaptic transmission genes including DNM1 cause epileptic encephalopathies. Am J Hum Genet. 2014;95:360-70.

42. Reuter MS, Tawamie H, Buchert R, Hosny Gebril O, Froukh T, Thiel C, et al. Diagnostic yield and novel candidate genes by exome sequencing in 152 consanguineous families with neurodevelopmental disorders. JAMA Psychiatry. 2017;74:293-9.

43. Anazi S, Maddirevula S, Salpietro V, Asi YT, Alsahli S, Alhashem A, et al. Expanding the genetic heterogeneity of intellectual disability. Hum Genet. 2017;136:1419-29.

44. Dumay-Odelot H, Marck C, Durrieu-Gaillard S, Lefebvre O, Jourdain S, Prochazkova M, et al. Identification, molecular cloning, and characterization of the sixth subunit of human transcription factor TFIIIC. J Biol Chem. 2007;282:17179-89.

45. Male G, von Appen A, Glatt S, Taylor NM, Cristovao M, Groetsch H, et al. Architecture of TFIIIC and its role in RNA polymerase III pre-initiation complex assembly. Nat Commun. 2015;6:7387. 
46. Borck G, Hog F, Dentici ML, Tan PL, Sowada N, Medeira A, et al. BRF1 mutations alter RNA polymerase III-dependent transcription and cause neurodevelopmental anomalies. Genome Res. 2015;25:155-66.

47. Jee YH, Sowada N, Markello TC, Rezvani I, Borck G, Baron J. BRF1 mutations in a family with growth failure, markedly delayed bone age, and central nervous system anomalies. Clin Genet. 2017;91:739-47.

48. Zhong F, Savage SA, Shkreli M, Giri N, Jessop L, Myers T, et al. Disruption of telomerase trafficking by TCAB 1 mutation causes dyskeratosis congenita. Genes Dev. 2011;25:11-16.

49. Walne AJ, Vulliamy T, Kirwan M, Plagnol V, Dokal I. Constitutional mutations in RTEL1 cause severe dyskeratosis congenita. Am J Hum Genet. 2013;92:448-53.

50. Frescas D, de Lange T. A TIN2 dyskeratosis congenita mutation causes telomerase-independent telomere shortening in mice. Genes Dev. 2014;28:153-66.

51. Anazi S, Maddirevula S, Faqeih E, Alsedairy H, Alzahrani F, Shamseldin HE, et al. Clinical genomics expands the morbid genome of intellectual disability and offers a high diagnostic yield. Mol Psychiatry. 2017;22:615-24.

52. Nahorski MS, Maddirevula S, Ishimura R, Alsahli S, Brady AF, Begemann A, et al. Biallelic UFM1 and UFC1 mutations expand the essential role of ufmylation in brain development. Brain. 2018;141:1934-45.

53. Colin E, Daniel J, Ziegler A, Wakim J, Scrivo A, Haack TB, et al. Biallelic variants in UBA5 reveal that disruption of the UFM1 cascade can result in early-onset encephalopathy. Am J Hum Genet. 2016;99:695-703.

54. Arnadottir GA, Jensson BO, Marelsson SE, ulem G, Oddsson A, Kristjansson RP, et al. Compound heterozygous mutations in UBA5 causing early-onset epileptic encephalopathy in two sisters. BMC Med Genet. 2017;18:103.

55. Muona M, Ishimura R, Laari A, Ichimura Y, Linnankivi T, KeskiFilppula R, et al. Biallelic variants in UBA5 link dysfunctional UFM1 ubiquitin-like modifier pathway to severe infantile-onset encephalopathy. Am J Hum Genet. 2016;99:683-94.

56. Low KJ, Baptista J, Babiker M, Caswell R, King C, Ellard S, et al. Hemizygous UBA5 missense mutation unmasks recessive disorder in a patient with infantile-onset encephalopathy, acquired microcephaly, small cerebellum, movement disorder and severe neurodevelopmental delay. Eur J Med Genet. 2018;pii:S17697212(18)30173-3.

57. Trump N, McTague A, Brittain H, Papandreou A, Meyer E, Ngoh $\mathrm{A}$, et al. Improving diagnosis and broadening the phenotypes in early-onset seizure and severe developmental delay disorders through gene panel analysis. J Med Genet. 2016;53:310-7.

58. Yang Y, Muzny DM, Xia F, Niu Z, Person R, Ding Y, et al. Molecular findings among patients referred for clinical wholeexome sequencing. JAMA. 2014;312:1870-9.

59. Tarailo-Graovac M, Shyr C, Ross CJ, Horvath GA, Salvarinova R, Ye XC, et al. Exome Sequencing and the Management of Neurometabolic Disorders. $N$ Engl $J$ Med. 2016;374:2246-55.

\section{Affiliations}

Sorina M. Papuc ${ }^{1,2} \cdot$ Lucia Abela $^{3,4,5} \cdot$ Katharina Steindl $^{1} \cdot$ Anaïs Begemann $\mathbb{1}^{1} \cdot$ Thomas L. Simmons $^{3}$. Bernhard Schmitt ${ }^{3,4}$. Markus Zweier ${ }^{1}$ - Beatrice Oneda' ${ }^{1}$ Eileen Socher ${ }^{6}$ - Lisa M. Crowther ${ }^{3}$ - Gabriele Wohlrab ${ }^{3}$.

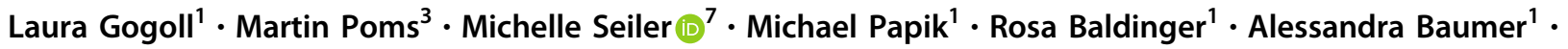
Reza Asadollahi $\mathbb{1}^{1}$. Judith Kroell-Seger ${ }^{8} \cdot$ Regula Schmid $^{9} \cdot$ Tobias Iff $^{10}$ - Thomas Schmitt-Mechelke ${ }^{11}$. Karoline Otten ${ }^{8}$ - Annette Hackenberg ${ }^{3}$ - Marie-Claude Addor ${ }^{12}$ - Andrea Klein ${ }^{13,14}$ - Silvia Azzarello-Burri ${ }^{1}$. Heinrich Sticht ${ }^{6} \cdot$ Pascal Joset $^{1} \cdot$ Barbara Plecko ${ }^{3,4,5,15,16} \cdot$ Anita Rauch $\mathbb{1}^{1,5,15,17}$

1 Institute of Medical Genetics, University of Zurich, SchlierenZurich 8952, Switzerland

2 Victor Babes National Institute of Pathology, Bucharest 050096, Romania

3 Division of Child Neurology, University Children's Hospital Zurich, Zurich 8032, Switzerland

4 CRC Clinical Research Center University, Children's Hospital Zurich, Zurich 8032, Switzerland

5 radiz-Rare Disease Initiative Zürich, Clinical Research Priority Program for Rare Diseases University of Zurich, Zurich 8032, Switzerland

6 Institute of Biochemistry, Friedrich-Alexander-Universität Erlangen-Nürnberg (FAU), Erlangen 91054, Germany

7 Pediatric Emergency Department, University Children's Hospital Zurich, Zurich 8032, Switzerland

8 Children's department, Swiss Epilepsy Centre, Clinic Lengg, Zurich 8000, Switzerland

9 Division of Child Neurology, Kantonsspital Winterthur,
Winterthur 8401, Switzerland

10 Municipal Hospital of Zurich Triemli, Zurich 8063, Switzerland

11 Division of Child Neurology, Children's Hospital, Lucerne 6000, Switzerland

12 Department of Woman-Mother-Child, University Medical Center CHUV, Lausanne 1015, Switzerland

13 Division of Paediatric Neurology, University Childerns Hospital Basel, UKBB, 4031 Basel, Switzerland

14 Division of Paediatric Neurology, Development and Rehabilitation, University Children's Hospital, 3010 Bern, Switzerland

15 Neuroscience Center Zurich, University of Zurich and ETH Zurich, Zurich 8057, Switzerland

16 Division of General Pediatrics, Department of Pediatrics, Medical University of Graz, 8036 Graz, Austria

17 Zurich Center for Integrative Human Physiology, University of Zurich, Zurich 8057, Switzerland 